\title{
Exploring Non-Conventional Sensorimotor Devices in a Virtual Bicycle Simulator
}

\author{
Raphael de Souza e Almeida (D) [ Pontifícia Universidade Católica do Rio de Janeiro | rsalmeida@tecgraf.puc-rio.br ] \\ Renato Cherullo (D) [ Pontifícia Universidade Católica do Rio de Janeiro | cherullo@tecgraf.puc-rio.br ] \\ Daniel Radetic (D) [ Pontifícia Universidade Católica do Rio de Janeiro | radetic@tecgraf.puc-rio.br ] \\ Greis Francy M. Silva-Calpa (D) [ Pontifícia Universidade Católica do Rio de Janeiro $\mid$ greis@tecgraf.puc-rio.br ] \\ Alberto B. Raposo (D) [ Pontifícia Universidade Católica do Rio de Janeiro | abraposo@tecgraf.puc-rio.br ]
}

\begin{abstract}
This work presents SimBike, a virtual bicycle simulator that uses non-conventional motor, sensory and sensorimotor devices to provide greater user involvement and comfort. SimBike aims to recreate the activity of riding a bicycle by exploring possibilities of electronic devices, such as making curves with the handlebar or body-weight, pedaling, braking, among others. It also offers feedback commonly expected by the rider, such as wind sensation varying according to speed and the sensation of trepidation on uneven terrain. The simulator was evaluated with 16 users, obtaining positive results regarding user experience, comfort, and immersion. As future work, we propose to improve some features to adapt the simulator for all users, regardless of their weight and height.
\end{abstract}

Keywords: Bicycle Simulator, Virtual Reality, Cyber Sickness, Non-Conventional Devices, User Experience

\section{Introduction}

Cycling is recognized as one of the means with the potential to promote health. Among its benefits, we can mention the regulation of cardiorespiratory and metabolic functions. These benefits can be observed even in the use of static bicycle in closed places Chavarrias et al. (2019). Chavarrias et al. (2019) suggest that indoor cycling has benefits for, among others, increasing aerobic capacity and reducing total cholesterol.

However, indoor cycling can reduce the positive experience that outdoor cycling can bring. With the technological advancements of various areas driven by Industry 4.0, new opportunities to develop better applications for both training and entertainment have become possible. Among them, several Virtual Reality (VR) simulators have been developed to recreate the activity of cycling, applied to different contexts, such as education Schulzyk et al. (2007), traffic safety training O'Hern et al. (2017), physical rehabilitation Pedroli et al. (2018), and sports training Pepper (2019).

Some studies such as Sun and Qing (2018) and Bolton et al. (2014), report that not all devices used contributed entirely to the realism of the activity. Freina and Ott (2015) suggest that for a complete sense of immersion, our five senses must be involved. Therefore, it is important to pursue solutions that provide different feedback modalities in order to create an user experience that is more comfortable, immersive, and realistic.

In the literature, we found that bicycle simulators usually employ behavioral interfaces to convey realism and immersion. A behavioral interface is one that "depends on a device that uses the motricity or perceptions of man resulting from his behavior in the real world" Fuchs et al. (2011). In this case, it means that the devices used on a bicycle simulator should allow the user to act during the experience as close as possible as $\mathrm{s} / \mathrm{he}$ would act when riding a real bicycle, while receiving the same set of sensory feedback.
When observing the basic movements of a cyclist during an usual bicycle ride (pedaling, applying the brakes, handling the handlebar, etc.), as well as the responses to these movements (acceleration, wind, vibrations), we can realize how complex it can be to separate each set of senses to arrive at each of the sensations experienced.

We developed a first version of our simulator, and from our observations, we identified the importance of providing feedback through different low-cost devices. That simulator consisted of a real bike attached to a training roller. We used a mouse attached to the bicycle frame to detect the movement of the handlebar. The rear wheel movement was detected using a hall effect sensor and four magnets attached symmetrically to the rear wheel. The DK2 version of the Oculus Rift HMD was used. In that experiment, we observed that experienced cyclists usually felt the most discomfort. In essence, they had a hard time staying balanced on top of the static bike, and sometimes, depending on their sensitivity, even had symptoms related to cybersickness.

Aiming to reduce this discomfort in a virtual simulator, we analyzed the behavior of a cyclist in the real world. We observed that when a cyclist performs a turn at high speeds, first $\mathrm{s} /$ he does a slight body movement, shifting the weight in the desired curve direction, and then changes the angle of attack of the front wheel through the handlebar. Both movements are subtle and happen in quick succession. Although our previous simulator did detect the handlebar's movement, it did not account for the weight shifting at all.

To tackle this limitation and improve the overall quality of the sensors used, we developed SimBike, a low-cost bicycle simulator that uses non-conventional motor and sensory devices. Among them, we used potentiometers that can be found on light control of a house room, and a motor for the haptic feedback that can be found in a PlayStation 3 controller. We also used weight load cells to capture weight distribution, extensively using Arduinos Arduino AG (2020). This study aims to identify how the devices in SimBike con- 
tribute to the user experience in the virtual simulator, including the level of immersion, realism, and cybersickness symptoms.

This paper is a reviewed extension version of the paper de Souza e Almeida et al. (2019). The remainder of this paper is organized as follows. In the next section, we present some related works. Next, we describe the proposed simulator and the devices used. Later, we detail the evaluation process of the simulator. Next, we present the results and discussions. Finally, we present the conclusions of this study.

\section{Related Work}

In this section, we describe the devices used in bicycle simulators from some studies in the literature; then, we mention possible solutions for gaps identified in these devices.

Bolton et al. (2014), developed a bicycle simulator where speed is captured by a device (Wahoo Kickr Power Trainer) attached to a bicycle. Data are sent to a mobile phone via Bluetooth, which sends the speed data to a Unity based game. The bicycle is not capable of steering, but the user gestures are detected using a Microsoft Kinect. The authors point out that although the interaction through Kinect provides great immersion, the high latency caused by the use of the Microsoft Kinect camera can cause risks of falling players. They noted that the simulator could increase immersion by increasing resistance when a user hits obstacles or by adding uphill and downhill levels with simulate grade, which allow them to provide levels to match specific fitness goals by simulating hill climbs, intervals, etc.

Ahmadpour et al. (2017), proposed a simulator using a tricycle and Google Street View imagery. On that work the participants took part of an experience in two conditions, randomly allocated: the Condition I, where User Interface consists of a static image of a scenic road, and the Condition II, where Open World depicts visual content from Google Street View imagery while the user is rides the tricycle. In Condition II motion detection occurs as a result of a sensor positioned on the rear wheel, while the change of direction occurs through an HMD control linked to the handlebar. The authors mentioned that almost half of the participants in the Condition I showed disinterest after a short interaction time, due to the static environment.

Rakhmatov et al. (2018), proposed a simulator that captures vibrations and speed from the cyclist's handlebar during a journey in different types of real terrain, generating texture models to be identified in a VR journey. The authors tested the simulator and they highlighted that the participants mentioned a lack of feedback from macro features from the road surfaces that harmed realism. They also found that it is required to use actuators to move the whole bicycle frame. Also, several participants reported that it is quite hard to focus on the feedback response during pedaling. They presume that for low velocities the vibrations of the chain mechanism where bigger than then device feedback. As future work, the authors intend to use rendering of macro features on a perturbation platform.

Sun and Qing (2018) explored unconventional motor devices to allow inputs and provide feedback in a simulator called ZouSim. The bicycle is composed of a small Trek 800 bike with a 13" women's frame and 26" wheels to accommodate riders. The ZouSim hardware to measure speed has three components: a rotation translator, a voltage conversion circuit, and an analog to digital converter. To acquire speed a dynamo is used. To measure braking a circuit involving a biasing voltage and a potentiometer was used. They conducted an experiment using the equipment. The authors tested four systems to measure the direction: video, using Microsoft Kinect sensor, mechanical, with a base that allows rotation and optical, with a light emitting diode or laser with a plastic lens low-resolution high-speed camera where The result of the processing is the determination of the amount of handlebar rotation Sun and Qing (2018). The authors noted that the centralization of bicycle software could be useful to combat steering drift and related problems. Also a vibration generator can be easily added to simulate movement due to imperfections in the road and interaction between tire and pavement.

Boulanger et al. (2017), developed MedBike, a low-cost bicycle simulator to support the rehabilitation of people with heart conditions. The MedBike simulator consists of patients cycling on a static bike while their vital signs were monitored. The authors used a linear potentiometer for the brakes, a fan for wind feedback, force sensors positioned on the handlebar to capture the attempt to change the direction, and a sensor positioned on the front wheel to capture the acceleration. The authors concluded that MedBike is easy to install, to test it's functionality better diagnostic tools should be developed. However, they did not report results related to devices used, such as the linear potentiometer and the wind sensation.

The results of these studies suggest that they complement each other and could provide an even more pleasant experience if combined. Another insight that we can realize was the different ways authors employed to capture users' attempts to change directions, each one exploring different strategies. Authors also related some issues with their current implementations, such as movement latency while making movements on the bicycle, bicycle steering restrictions, difficulties such as users do not perceiving haptic feedback from ground texture and disinterest from participants after a short interaction time, due to the static environment.

From the study by Bolton et al. (2014), we highlighted the mechanism to capture the speed, it is captured by the movement of the pedals and inserted into the simulation. However, the absence of steering may compromise immersion. The steering solution proposed by Ahmadpour et al. (2017) brings a low latency result and, combining with Google Street View imagery, could deliver a good ridding experience. We also highlighted the haptic feedback proposed by Rakhmatov et al. (2018), which could bring more realistic experience with the feeling of ground texture. Other possibility that emerges with haptic feedback is the crash sensation that the devices attached to the bicycle could bring by the time the user touch with the bicycle in the wall.

The concepts obtained from ZouSim devices Sun and Qing (2018), like using a potentiometer to obtain the braking intensity, exploring a dynamo to obtain a gradual acceleration and vibration generator to simulate road imperfections, could be implemented with low-cost similar devices like a 
mouse replacing the dynamo on acceleration and $1.5 \mathrm{~V}$ motor to generate the vibration and providing feedback. The study by Boulanger et al. (2017) also included potentiometers and used a fan to the generation of wind. Although the authors did not report the contribution of those devices, we believe that they can contribute with user immersion which could bring more comfort to the simulation.

Building on the strengths and avoiding the shortcomings of the studies above, one can design a simulation that provides solutions to those issues while preserving the immersive aspects observed. And so We developed the devices on SimBike, as described below.

\section{Proposed Bicycle Simulator}

We developed SimBike, a virtual simulator that uses unusual motor and sensory devices to improve a more immersive experience. SimBike allows a user wearing an HMD device to ride in a virtual track while sitting on a real bike. The bike is connected with a computer that runs the simulated environment. The user-simulator interaction is similar to use a real bike, using the pedals, the handlebar, brakes, and leaning from side to side.

SimBike was developed on the Unity3D game engine Unity Technologies (2019), and incorporates a number of custom-made, Arduino-based devices. The general requirements considered in the development of our simulator and the implementation process are described below.

\subsection{General Requirements}

According to Fuchs et al. (2011), behavioral interfaces aim to allow the users to act on the virtual world in the same manner that they would act on the real world while performing some specific activity. For they, behavioral interfaces can be divided into sensory interfaces and motor interfaces.

Sensory interfaces provide feedback to the user of what is happening in the virtual environment (e.g. computer screens, headphones, haptic feedback devices). Low latency and synchronism are essential factors that should always be prioritized, under penalty of breaking immersion or causing cybersickness. In some cases, feedback is easily perceived by the user. For instance, any small change in the virtual bicycle's position will be easily noticed by the user since its point of view in the virtual world will change. Motor interfaces are those capable of detecting the user's actions (e.g. gamepads, Microsoft Kinect sensor). It follows that sensorimotor interfaces are capable of both detecting user actions and providing sensory feedback.

Those interfaces are implemented on top of physical devices. For this study, the motor devices are built to be as transparent as possible to the user, in the sense that the user operates the bicycle just like any other regular bicycle. Low latency was also a top priority when selecting the motor devices, since here every user action can lead to changes in speed and position, which are easily perceived by the user.

Notice that it can be quite hard to detect some user actions or some qualities of detected actions, so there is always a trade-off involved. For example, we are only interested in the user's arms and legs movements as long as it affects the handlebar, brakes, pedals, or moves the user's center of mass. Precise positioning of elbows and knees is not deemed important and is not trivial to implement either.

\subsection{Implementation}

SimBike consists of a bicycle, bolted to an ALTMAYER Sports training roller Altmayer Sport (2019). The base of this roller was reinforced with two 1.5 meter metal bars, attached to each side of the base. Several additional motor and sensory devices are attached to or placed around the bicycleand-roller assembly, as shown in Figure 1.

The development of these devices was largely made possible by the creation of a library to manage all the communication ports (COM) used to connect the Arduinos to the computer where the simulation is run. The library standardizes the discovery and handling of connection and disconnection of devices at runtime, facilitating the use of several Arduinos simultaneously.

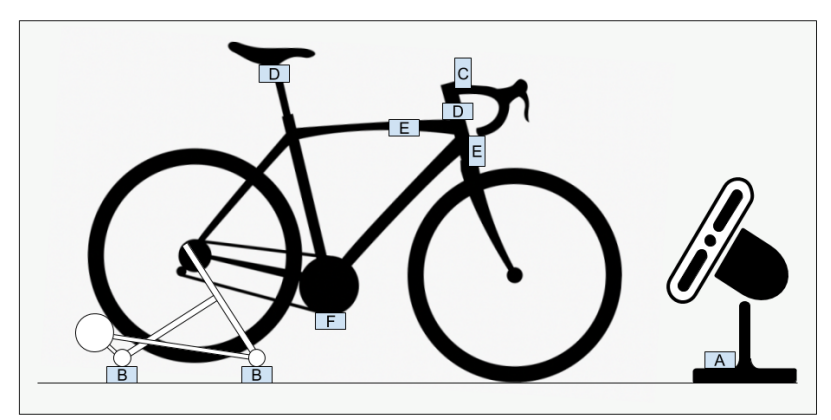

Figure 1. Schematic image of all devices included in the SimBike Simulator: A) A fan used for wind feedback. B) Load cells used to estimate the user's center of mass. C) A VR controller used to measure the handlebar's forward direction. D) $1.5 \mathrm{~V}$ electric motors that provide haptic feedback. E) Brakemeasuring potentiometers. F) Mouse measuring the rotation of the central axle.

We developed the following features in SimBike: detection of acceleration (low latency pedal movement detection), braking (proportional to the pressure exerted on brakes), turning (change of direction based on both the direction the body is leaning and the handlebar direction), artificial wind feedback (to contribute to the sensation of speed), and impacts feedback (vibration resulting from contact with the terrain and impact response). The implementation of these features is described below.

\subsubsection{Acceleration}

In the real world, bicycle acceleration is achieved by applying force to the pedals, so acceleration in the simulated environment must also be proportional to the movement of the pedals. Measuring this requires a sensor that can detect the direction and speed of the movement, with as little latency as 
possible. Thus, we chose to adapt a mouse to measure the rotation of the central axle, allowing us to measure movement in both directions with very low latency.

We attached the mouse to a surface that itself was attached to the bicycle's frame to capture the movements of a protective cover of the central axle, as shown in Figure 2. It is worth noting that the value we are measuring is proportional to the angular speed of the central axle, so we have no way of determining neither the precise position of the pedals nor the torque that is being applied by the cyclist.

Thus, to use the movement values provided by the mouse, it is first necessary to determine how many units of distance it reports for each degree of rotation of the pedal, which can be easily measured. However, it is important to note that this measurement depends on the mouse model and the operating system settings.

One aspect to consider is the small resistance of the pedals since the rear wheel of the bicycle is raised by the safety bars. In some cases, if one maintains the torque $100 \%$ in the rear wheel, it can generate a great amount of torque in the virtual bike due to the physics applied in the simulation, enough to lift the front wheel or even flip the bicycle backward. To avoid situations like that we distributed the applied torque in a way that $30 \%$ goes to the front wheel and $70 \%$ goes to the rear wheel.

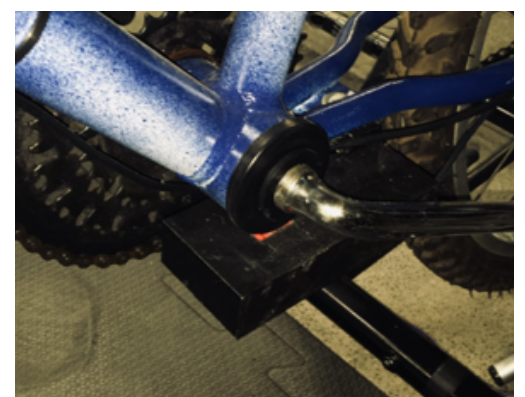

Figure 2. Mouse positioned to capture the axle's protection cover rotation.

\subsubsection{Braking}

The requirements adopted to develop the device for braking detection were the following: its latency should be as low as the acceleration detection device, it should allow the detection of different levels of brake intensity, and be independent of the speed detection mechanism.

Therefore, we used linear potentiometers attached to the bicycle's brake cables. We used two $10 \mathrm{k} \Omega$ modules, one for the rear brakes and another for the front brakes (letter $\mathbf{e}$ in Figure 1). Figure 3 shows the modules installed in their digitally printed cases.

The values of the potentiometers are read on demand through two analog pins on an Arduino and sent to the PC as two integer values between 0 and 64 (both inclusive), where we perform the dead zone calculations. For this, we use the function below for both brakes:

$$
f(s)= \begin{cases}0, & s \leq z \\ \frac{s-z}{M-z}, & s>z\end{cases}
$$

where $f(s) \in[0,1]$ is the calculated braking intensity, $s \in$

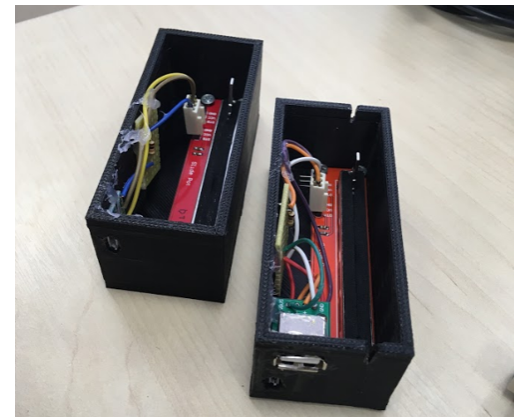

Figure 3. Linear potentiometers used to measure braking intensity.

$[0, M]$ is the sensor read value, $z \in[0, M)$ is the dead zone length, and $M$ is the maximum value that the sensor can report. In our case, $z=5$ and $M=64$.

This function has two important properties: it is continuous at $s=z$ and its image is the entire $[0,1]$ range. Compared to a naive implementation, it allows greater precision in weak braking situations. The calculated braking intensity is then multiplied by the maximum torque of the brakes and applied to the respective wheel.

\subsubsection{Turning}

When observing a cyclist during an usual bicycle ride, we identified that as the bicycle's speed increases, the shifting of the cyclist's balance has a more significant role in establishing the bicycle's turning rate than the handlebar itself. At low speeds, the cyclist shifts its weight seeking to maintain the balance, while the handlebar become predominant in determining the direction where the bicycle will go. Our simulator intends to reflect these properties in the virtual environment.

Thus, we attached an HMD controller (HTC Vive Controller) to the handlebar to determine its direction (letter $c$ in Figure 1) with precision and low latency. This setup allows us to position the user's point of view in the virtual environment correctly in regards to the virtual bicycle so the user sees the virtual bicycle where the real bicycle is.

The HMD software provides us a "play area" with the virtual positions of the HMD and its controllers, where they are correctly positioned relative to one another. Assuming that the real handlebar is facing forward, if we move this "play area" to match the position and orientation of the virtual controller in the center of the virtual handlebar, the user's point of view in the virtual environment will be correctly positioned relative to the virtual bicycle.

When using only the handlebar to control the bicycle, we observed that the higher the speed, the harder it becomes to maneuver the bicycle: any small movement of the handlebar leads to oversteering. To solve this problem, we implemented a curve to attenuate the effective angle of the handlebar based on the bicycle's speed. This also reinforces the desired behavior outlined above where the handlebar should be less important at high speeds.

In order to preserve the matching between the real handlebar (and thus the user's hands) and the virtual handlebar, we continue to draw the virtual handlebar in the expected position, the attenuated value is only used in the physics simulation. 


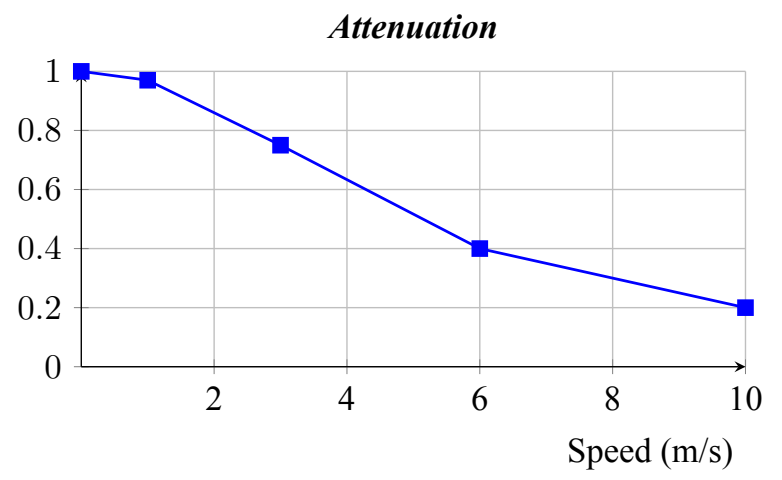

Figure 4. Handlebar attenuation curve as a function of bicycle speed, empirically obtained.

We adopted the convention that the handlebar angle would be represented in degrees, with values in the range $[-90,90]$, where zero is the longitudinal direction of the bicycle, with values increasing clockwise. Then, to perform the attenuation, we multiplied the angle reported by the HMD controller by the attenuation factor that corresponds to the current speed of the bicycle in the curve in Figure 4.

In order to measure the lateral shifting of the cyclist's balance, four load cells, with the capacity to hold up to $50 \mathrm{~kg}$ each, were distributed between the ends of the metal bars attached to the training roll, with two on each side (see purple circles in Figure 1).

The load cells were all connected to the same HX711 amplifier module. This module does not report the weight above the load cells directly; instead, it amplifies the electrical signal coming from them, requiring further calibration. We inverted the polarity of the load cells in one side of the bicycle, so the module was actually reporting the difference between the signals coming from each side of the bicycle: lower values when the weight on the left-hand side was higher than the weight on the right-hand side, and higher values on the reverse situation.

Additionally, the module was configured to report data at a frequency of $80 \mathrm{~Hz}$, which is crucial since the reported value is very noisy, requiring us to average 20 consecutive measurements to obtain a stable value. From this point forward, we will always be referring to this average.

Since the weight and posture of each user can be different, the calibration process consisted of storing the value $C$ obtained when the user is centered on the bicycle and, from then on, maintaining the minimum and maximum values measured so far, respectively $m$ and $M$. To obtain the normalized user balance input, we apply the following function to value $s$ read from the sensor each frame:

$$
b(s)= \begin{cases}\frac{s-C}{C-m}, & s \leq C \\ \frac{s-C}{M-C}, & s>C\end{cases}
$$

With $b(s) \in[-1,1]$, the value -1 achieved when the user shifts his weight to the left the most, 0 when the user is centered in the bicycle, and 1 when his weight is shifted to the right the most. We then adjust this value to account for a centered dead zone of length 0.05 to each side, using a function similar to (1) used for the brakes. This procedure allows the system to adapt itself dynamically to each user.

Finally, to combine the balance input with the handlebar input, when the user is shifting his weight to the same side on which the handlebar is turned to, we multiply the handlebar effective angle by the factor below:

$$
1+\|e\| \cdot B F(v)
$$

where $e \in[-1,1]$ is the balance input and $B F$ is the balance factor that corresponds to the current speed $v$ of the bicycle in the curve (Figure 5). Naturally, when the user is in balance, $e=0$ and so there is no influence of the balance in the bicycle's direction. The same happens when the bicycle is stopped, since $B F(0)=0$. Since $B F$ is a growing function, we have the desired behavior where the balance influence grows with speed.

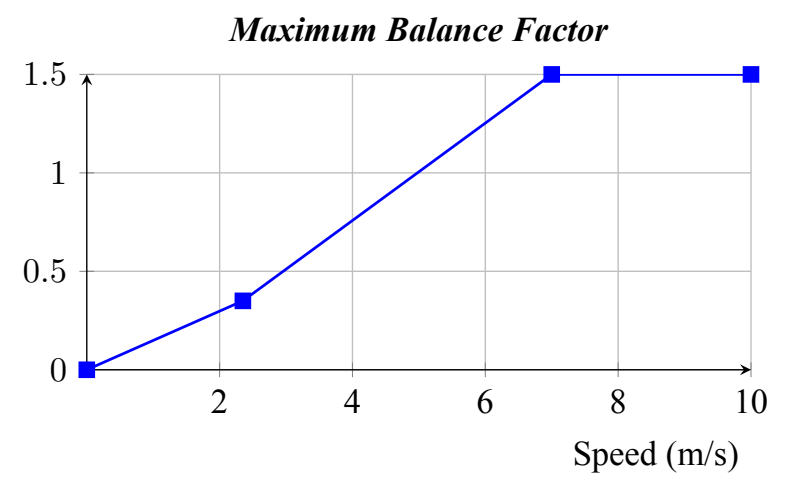

Figure 5. Maximum balance factor curve, as a function of the bike's speed.

\subsubsection{Artificial Wind}

We employ a fan to provide more feedback to the user, involving another sense into the experience. It's fundamental to break the sense of inertia felt by the cyclist as the bike speeds up, since $\mathrm{s} /$ he is not really moving forward, as well as matching the sensation of movement inferred from the visual cues. The main requirements were that the solution should be silent, so as not to break immersion, and allow some level of control over the wind speed, so it can be proportional to the speed of the bicycle in the simulation.

Thus, we used a $40 \mathrm{~cm}$ domestic fan, due to the ease of acquisition and silence of operation, controlled by an Arduino, which receives from the $\mathrm{PC}$ the relative power on which the fan must operate.

The implemented circuit consists of a zero detector and an optocoupler connected to a power outlet, so that we can detect the beginning of each wave of the alternating current, and control in a binary way the passage of energy from the electrical network to a female outlet where we connect the fan, as seen in Figure 6.

The first implementation of the fan control software was based on pulse width modulation, with the period equal to $\frac{1}{60} s$ synchronized to the main power frequency using the zero counter, but the behavior of the fan was not satisfactory for duty cycles below approximately $80 \%$ : besides the speed reached by the fan being low, it vibrated and made a lot of noise.

Instead, the refined software is based on the premise that we must always supply the fan with full periods of the alternating current. At the beginning of each period, we decide 


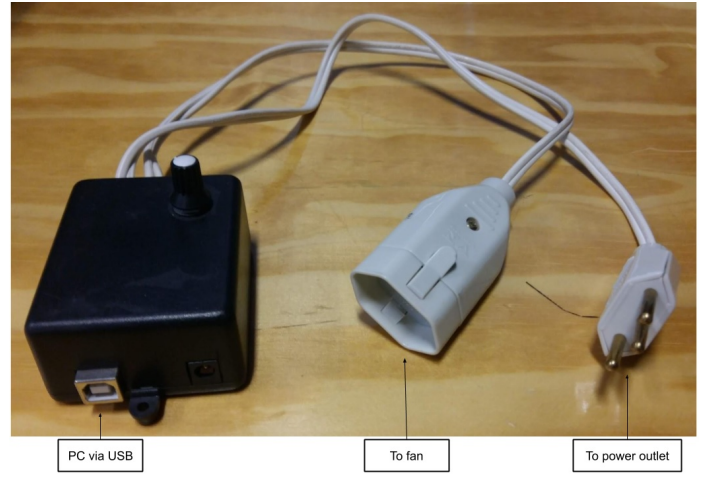

Figure 6. Arduino that controls the fan.

whether the fan will remain on based on the relative power requested by the $\mathrm{PC}$, represented by an integer between zero and 60 . The algorithm is simple and resembles an integer division by successive subtractions reusing the remainder. This resulted in more consistent and quieter fan behavior, in duty cycles ranging from $10 \%$ to $100 \%$.

One of the greatest challenges of using a fan as a feedback device in VR experiences is managing its latency. Even if all control systems are exemplary in this regard, a domestic fan can take a long time to reach the desired speed and there is no way to brake it either. To mitigate this problem, at the beginning of each experience the fan is turned on at full power for one second, and then we never let the power drop below $10 \%$.

\subsubsection{Impacts and Terrain Irregularity}

Another kind of feedback that's very perceivable when cycling is related to collisions and contact with the terrain. Clearly, those feedback should not be extreme, but their absence could cause discomfort since cyclists have a set of expectations in this regard. To implement this, two 5V DC electric motors with eccentric weights attached to their axes were used, one positioned under the seat and another on the handlebar (see letter $d$ in Figure 1), both controlled using an Arduino.

These motors were used to vibrate the bicycle the higher its speed, increasing linearly to, and being capped at, $80 \%$ of its maximum speed when the bike was at $5 \mathrm{~m} / \mathrm{s}$, transmitting the sensation caused by the contact of the bicycle's tires with the terrain's irregularities. Crash feedback consisted of a characteristic sound and the vibrating of the haptic feedback motors at maximum speed for one second.

\section{Evaluation}

We conducted an empirical evaluation of our simulator, aiming to identify if the proposed devices contributed to the user's presence and the reduction of cybersickness symptoms. We comparatively evaluated two modes, both using the bicycle devices, named: "Handlebar mode" and "Balance mode", described as follows.

The Handlebar mode considers input from all available devices, except the data from the load cells. And so, users can only change directions by moving the handlebar, while the user's weight distribution is completely disregarded. All feedback devices are also employed.
The Balance mode explores all available devices to bring the full experience of our simulator, combining the input from the handlebar with the input from the load cells, as described in section 3.2.3, allowing the user to influence the bicycle steering angle by shifting his/her body weight. Again, all feedback devices are employed.

In both modes, the user travels through a track that is similar to the letter " $\mathrm{B}$ ", containing a narrowing in its last curve, as shown in Figure 7. The track requires movement in both directions (right and left) aiming to explore the bicycle's devices. The narrowing of the last curve intends to force the participant to reduce the speed using the brakes, requiring precise movements. If the user does not succeed and ends up colliding, the impact through vibration on the bicycle must be perceived. A stopwatch was placed on the bicycle handlebar to motivate users to reach speeds suitable for the use of all sensors and finish the circuit in a short time.

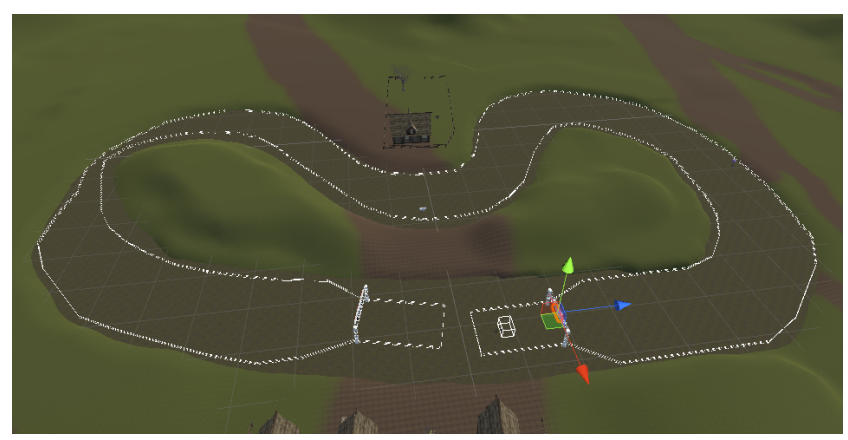

Figure 7. Virtual track traveled by users in the experiment.

\subsection{Participants}

Sixteen participants (13 males and 3 females - ages between 19 - 43 years old, with average weight $82.6 \mathrm{~kg}$ and average height $1.76 \mathrm{~m}$ ) took part in the tests. Each participant has a different level of experience in both VR and cycling.

We categorized participants by subgroups aiming to identify whether specific common characteristics influence their experience using the simulator: individuals over $90 \mathrm{~kg}(\mathrm{P} 1$, P5, P8, P9, P13, P14, P15), individuals under 60kg (P10, P11, $\mathrm{P} 16)$, individuals with height above $1.80 \mathrm{~m}$ (P5, P6, P7, P8, $\mathrm{P} 13, \mathrm{P} 14)$, individuals with more experience in VR (P4, P6, $\mathrm{P} 8, \mathrm{P} 14)$, and cycling (P1, P2, P3, P4, P15). Only one of the participants (P16) is still learning to ride a bicycle.

It should be noted that the weight $(82 \mathrm{~kg})$ and height $(1.70 \mathrm{~m})$ from the developer of our algorithm were considered as a reference for the initial calibration of the load cells, responsible for capturing the weight distribution by the bicycle.

\subsection{Procedure}

Tests were performed at the Laboratory of the Tecgraf Institute/PUC-Rio. The participants signed a consent form, and they could interrupt the experiment whenever they wished. In each test session, one user participated, accompanied by the researcher in the role of evaluator, providing support if necessary. 
Before each test, the participants received an explanation of the simulator concept and kinematics. They answered a pre-test questionnaire that addresses aspects such as frequency of cycling, use of VR equipment, age, height, and weight.

Each test consisted of performed the previously prepared track (Figure 7). The same track was used for both modes. Half of the participants first performed the Balance mode and, subsequently, the Handlebar mode. The other half of participants started with the Handlebar mode, ending with the Balance mode. The choice for which mode each participant started was random, in order to make possible attempts by subsequent users to obtain information difficult.

For each test, the participant took his/her place on the bicycle and placed the HMD device on his/her head. Both the HMD device and the participant's position on the bicycle were calibrated before each test. Each test session lasted a maximum of 15 minutes, including an explanation of SimBike, the pre-test questionnaire, the test, and the completion of the post-test questionnaires.

\subsection{Data Collection}

The data were collected by field notes performed during the tests, and questionnaires. We also analyzed the data generated by the simulator. We used two cameras to record the session tests. The cameras were installed in different angles to capture the user's reactions during the tests.

The field notes were obtained by direct observation and using the think-aloud technique. Participants were asked to report their positive and negative feelings either during or after using the simulator. We correlated these reports with the data collected in the simulator.

A pre-test questionnaire was applied once for each participant, collecting particular data from the user, like VR experience, bicycle experience, height and weight. After each experiment, the participants answered three questionnaires: one about the simulator in general (from here on called the post-test questionnaire), the Igroup Presence Questionnaire (IPQ), and the Simulator Sickness Questionnaire (SSQ).

The SimBike-specific post-test is a 10 -item 7 -scale questionnaire used to evaluate the user experience using the simulator in both the balance mode and Handlebar mode. Through that questionnaire, we identified aspects such as ease of use, joyfulness, immersion, and realism in the virtual environment according to the provided elements - artificial wind, steering, terrain contact, collision, and braking.

We used IPQ Schubert et al. (2001) to measure sense of presence experienced by user in the virtual simulator. IPQ uses a 7-point Likert scale to assess three axes: spatial Presence (the feeling of being physically present in the virtual environment), engagement (measuring user level of involvement and interest in the experiment), and realism (measuring the subjective experience of realism in the virtual environment).

Finally, we applied the SSQ questionnaire. SSQ measures cybersickness symptoms. It includes a set of questions asking for the severity of each symptom on a scale of 0-3 (0= "none", 1 ="slightly", 2 ="moderate", and 3 ="severe"). It should be noted that we used an translated version Carvalho et al. (2011) of the SSQ Kennedy and Frank (1985).

After the experiment, the simulator system generates data about it, such as speed, handlebar angle, simulation time, weight distribution intensity, among others. These data were analyzed and compared with the users' reports obtained through the questionnaires and think aloud. The results are described below.

\section{Results}

The following results are presented according to the aspects evaluated in the questionnaires and data generated by the simulator.

\subsection{Think-Aloud and SimBike-Specific Ques- tionnaire}

Based on the information collected during the think-aloud, we identified features in SimBike that provided a positive experience for most of the participants regardless of the mode used.

Overall, $56 \%$ of the participants reported during the test that riding a bicycle on the simulator was pleasant and easy to use. Also, $81 \%$ reported that the artificial wind contributed favorably to their immersion, mainly in the high-speed parts of the track in both modes.

We verified that the participants P4, P6 and P10, who did not report on the wind, had either lower speeds overall or stressing difficulty when maneuvering, according to their respective speed and steering plots. Participant P3, an experienced cyclist, mentioned that he had difficulty adapting to Handlebar mode direction changes, adding that the sensation of making a curve is pleasant on balance mode, but it is not well calibrated.

Regarding the SimBike-specific post-test questionnaire results, Figure 8 and Figure 9 show the average values reported by participants after the first and second experiment, respectively. Participants that used the Balance mode in the first experiment are the same ones who used the Handlebar mode on the second experiment, and vice-versa.

Since our sample size is of only 16 participants split in two groups, we cannot make absolute claims about our results. Still, we'll raise some observations that could lead to further investigations and insights.

Generally, "Wind Perception" and "Wind contribution to immersion" received high marks across the experiment, reflecting what the users reported during the think-aloud.

In the question entitled "Steering realism", graduated from 1 (nothing like a real bicycle) to 7 (realistic), as shown Figure 8 , the Balance mode seems to have some advantage over Handlebar mode, scoring an average of 4.13 against 3.25 after the first experiment. That didn't hold after the second experiment, with Balance mode scoring 3.38 against 5.0 in Handlebar mode (Figure 9).

About the "collision sensation" question, graduated from 1 (didn't feel) to 7 (really felt), participants evaluated in the Balance mode (value 4.75) and Handlebar mode (value 5.13) 


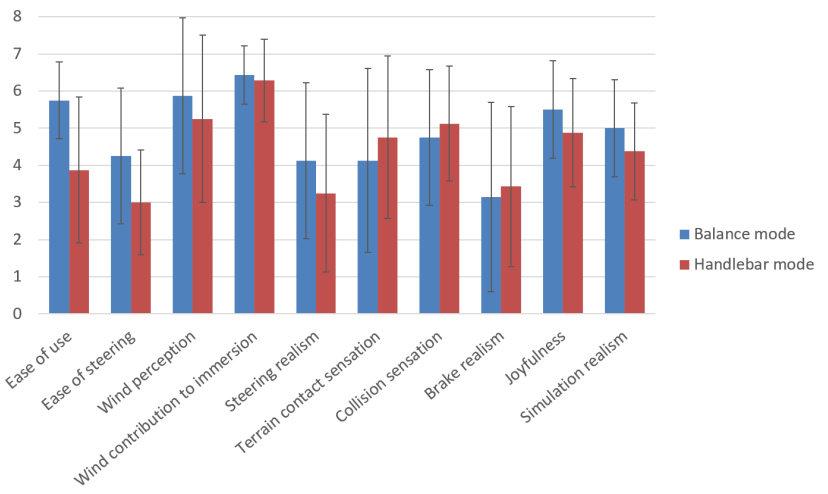

Figure 8. Averages values reported by participants after the first experiment through the SimBike-specific questionnaire. Participants using the Balance mode (blue bars) and participants using the Handlebar mode (red bars).

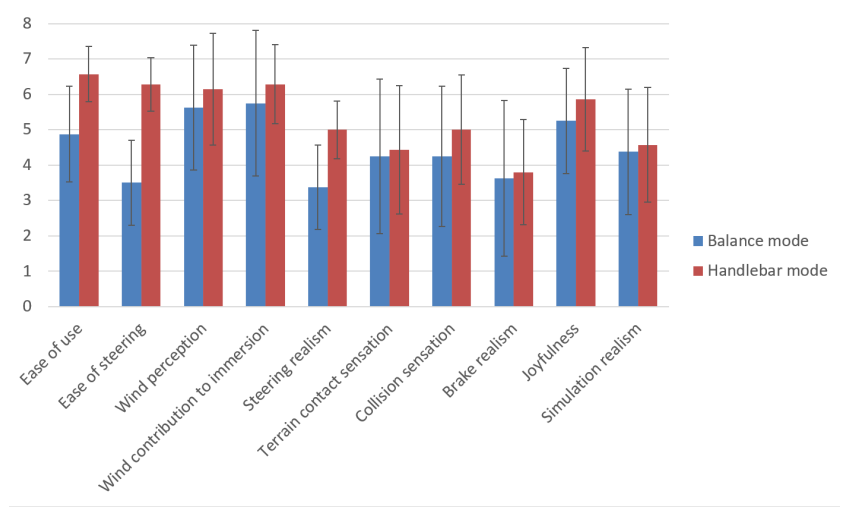

Figure 9. Averages values reported by participants after the second experiment through the SimBike-specific questionnaire. Participants using the Balance mode (blue bars) and participants using the Handlebar mode (red bars).

after the first experiment (Figure 8). At the second experiment (Figure 9), the participant evaluated with higher scores for Handlebar mode that for the Balance mode (5.0 versus 4.25).

Regarding to "ease of steering", evaluated from 1 (very hard) to 7 (very easy), after the first experiment (Figure 8), Balance and Handlebar mode scored 4.25 and 3.0, respectively. On the second experiment, Balance and Handlebar modes scored respectively 3.5 and 6.29.

\subsection{Sense of Presence, Engagement and Real- ism}

The sense of presence in the virtual simulator, including spatial presence, engagement and realism, was measured by the IPQ questionnaire. Figures 10 and 11 show similar grades in both the Handlebar and the Balance mode.

Spatial presence (Figure 10 and 11), which measures the sense of being physically present in the virtual environment Slater and Wilbur (1997), graduated from 1 to 7 , obtained mean values of 5.4 and 5.37 in Handlebar mode and Balance mode in the first experiment and 5.71 and 4.88, respectively, in the second experiment. There we can observe that participants that experienced the Balance mode first awarded higher grades than those that experienced the Handlebar mode first. Later, the users that experienced the Handlebar mode first awarded higher grades to the Balance mode on the second experiment. Particularly, four participants (P1, P7, P12, and $\mathrm{P} 13)$ related that they had the sensation of presence in both

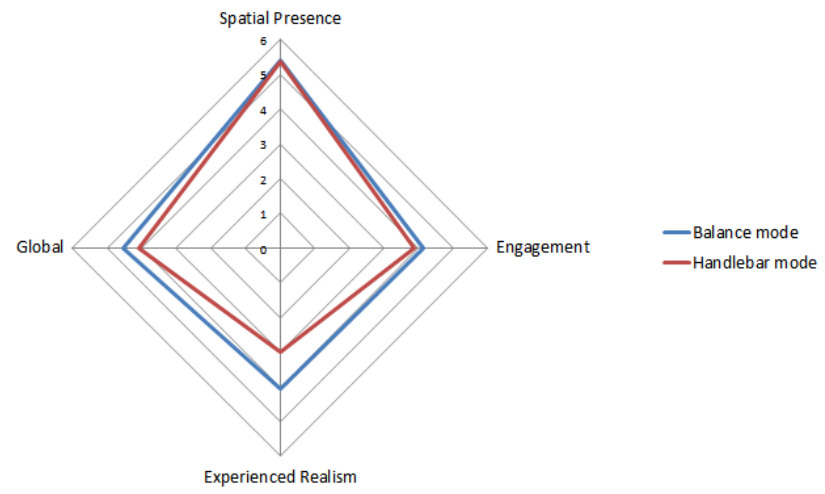

Figure 10. Mean values for the IPQ questionnaire in the Balance mode (blue bars) and Handlebar mode (red bars) at the first experiment.

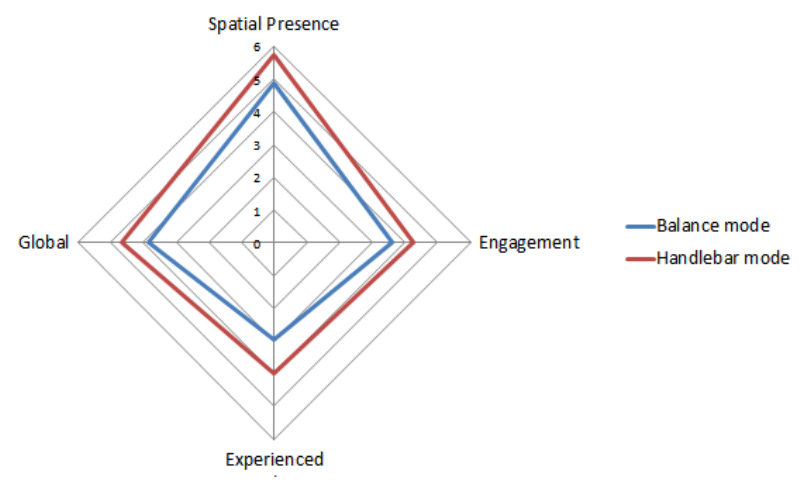

Figure 11. Mean values for the IPQ questionnaire in the Balance mode (blue bars) and Handlebar mode (red bars) at the second experiment.

modes. Regarding the engagement in the virtual environment, the obtained mean values on the first experiment were 4.14 in Balance mode and 3.85 in the Handlebar mode, while on the second experiment the values were 3.6 and 4.25 respectively. The participants P10 and P14 highlighted that the experience timer instigated them to accelerate to complete the course as fast as possible. This aspect involved them in the virtual experience.

The experienced realism category, measuring the subjective experience of realism in the virtual environment, was the less graduated, obtaining, in the first experiment, in Balance mode and Handlebar mode, grades 4.07 and 3.0 respectively and, at the second experiment, grades 2.96 and 4.0, again respectively. One aspect that could cause that lower grade was the Level of Detail (LOD) technique and effects of a short far plane in the eye cameras, as reported by a participant. This response, however, indicates a dissociation between realism and presence, showing that users were able to finish the course and engage their surroundings despite it's visual shortcomings.

Mean values obtained for global presence perception (Figure 10 and 11) were 4.53 and 4.07 for Balance mode and Handlebar mode, respectively, in the first experiment, and 3.81 and 4.65, again respectively, in the second experiment. We identified that participants shorter than $1.65 \mathrm{~m}$ reported higher values for Global presence. That fact can be associated with the user's comfortable positioning on the bicycle, that can better suited to shorter people. 


\subsection{Cybersickness Symptoms}

We used the SSQ questionnaire to measure cybersickness symptoms. Results show that three participants reported some slight or moderate symptoms after using the simulator (Tables 1 and 2). It was identified that symptoms of discomfort were reported mainly by participants with a height greater than $1.80 \mathrm{~m}(\mathrm{P} 6, \mathrm{P} 7$ and $\mathrm{P} 14)$. They reported a moderate degree in three or more symptoms, in particular to "difficulty focusing", "dizziness with eyes open" and "dizziness with eyes closed". The remainder of the participants reported fewer symptoms of discomfort in Balance mode than in Handlebar mode. Also, these symptoms were below moderate degree in both modes.

Table 1. Results of the SSQ questionnaire. Total of participants who reported symptoms after the experiment using the "Handlebar mode."

\begin{tabular}{lcccc}
\hline \hline Symptoms & None & Slight & Moderate & Severe \\
\hline General discomfort & 16 & 0 & 0 & 0 \\
Fatigue & 13 & 3 & 0 & 0 \\
Headache & 13 & 3 & 0 & 0 \\
Eyestrain & 15 & 1 & 0 & 0 \\
Difficulty focusing & 16 & 0 & 0 & 0 \\
Increased salivation & 16 & 0 & 0 & 0 \\
Sweating & 16 & 0 & 0 & 0 \\
Nausea & 16 & 0 & 0 & 0 \\
Difficulty concentrating & 16 & 0 & 0 & 0 \\
Fullness of head & 13 & 2 & 1 & 0 \\
Blurred vision & 15 & 0 & 1 & 0 \\
Dizzy (eyes open) & 12 & 2 & 0 & 0 \\
Dizzy (eyes closed) & 15 & 1 & 0 & 0 \\
Vertigo & 15 & 0 & 1 & 0 \\
Stomach awareness & 16 & 0 & 0 & 0 \\
Burping & 16 & 0 & 0 & 0 \\
\hline \hline
\end{tabular}

Table 2. Results of the SSQ questionnaire. Total of participants who reported symptoms after the experiment using the "Balance mode."

\begin{tabular}{lcccc}
\hline \hline Symptoms & None & Slight & Moderate & Severe \\
\hline General discomfort & 14 & 2 & 0 & 0 \\
Fatigue & 16 & 0 & 0 & 0 \\
Headache & 15 & 1 & 0 & 0 \\
Eyestrain & 15 & 1 & 0 & 0 \\
Difficulty focusing & 14 & 1 & 1 & 0 \\
Increased salivation & 15 & 1 & 0 & 0 \\
Sweating & 15 & 1 & 0 & 0 \\
Nausea & 15 & 1 & 0 & 0 \\
Difficulty concentrating & 15 & 1 & 0 & 0 \\
Fullness of head & 14 & 2 & 0 & 0 \\
Blurred vision & 15 & 0 & 1 & 0 \\
Dizzy (eyes open) & 14 & 1 & 1 & 0 \\
Dizzy (eyes closed) & 14 & 2 & 0 & 0 \\
Vertigo & 15 & 1 & 0 & 0 \\
Stomach awareness & 15 & 1 & 0 & 0 \\
Burping & 16 & 0 & 0 & 0 \\
\hline \hline
\end{tabular}

We observed that the participants had fewer symptoms of discomfort in the Balance mode than in the Handlebar mode, which may have occurred as a result of the user's effort to make the curve leaning to the desired direction, corresponding, in part, to the expectations of his vestibular system. Reports from participant $\mathrm{P} 4$ corroborate this observation. P4 reported that changing direction in Handlebar mode is strange, as he is used to shifting his body weight to make the turn. He is among the participants with experience in VR who experienced two or more symptoms in Handlebar mode.

\subsection{Results from Speed Data}

For all eight participants weighing more than $90 \mathrm{~kg}$, the speed plots show sharper peaks in higher speed sections, when the weight distribution influence is greater. For those participants weighting less than $60 \mathrm{~kg}$, we observed a graph with smoother lines both at low and high speeds.

As an example, Figures 12, 13 and 14 shows data from participants P15 (119 kg) and P10 (49 kg), in items a) and b) respectively. Figure 12 plots speed as a function of time, and Figure 13 plots the steering angle as a function of time, with positive values for right turns and negative values for left turns. The six color-coded dots observed in Figures 12 and 13 represents distinct moments in the same run of P15 and also P10, as it can be seen in Figure 14 where we portray at a) P15's trajectory through the track and b) P10's as well.

We observed that at the beginning of the balance mode experiment (i.e., the yellow and brown dots in Figures 12, 13 and 14), the acceleration and speed of the participant P15 (Figure 12 a) during a sudden pair of opposing signed spikes in steering angle (Figure $13 \mathrm{a}$ ). Watching the respective moment in the video footage, we identified that those spikes happen when both of them loses control of the bicycle. It was an attempt to make a turn met with exceeding response originating from the weight transfer, resulting in a corrective maneuver. This fact is a direct consequence of the weight factor configuration's sensitivity. This behavior can be verified in the first turn to the left (yellow and brown dots in the Figure 14) and the narrowing at the end (green and blue dots in the Figure 14).

Regarding participant P10, Figure $12 \mathrm{~b}$ ) and Figure $13 \mathrm{~b}$ ) show that those spikes happened at the beginning of the exercise similar to the P15's experiment and, after those spikes, the rest of the tracking was without sudden movements. We can understand that, in the first moment those points were ones that weight factor influenced by the adaptation of the user and, in the narrow point, the need for precision. We can identify that by the time there are higher speeds the higher probability to have more spikes.

We found no significant visual distinction between Handlebar mode and Balance mode for participants weighting less than $60 \mathrm{~kg}$. It contrasts with participants weighing above $90 \mathrm{~kg}$, which have difficulties to control the turning rate in the Balance mode. Both issues are built on the elevated sensitivity of the load cells and of the implemented model.

\section{Discussion}

The results obtained show that our proposed simulator has characteristics, such as easy to use, easy to steering, and wind 


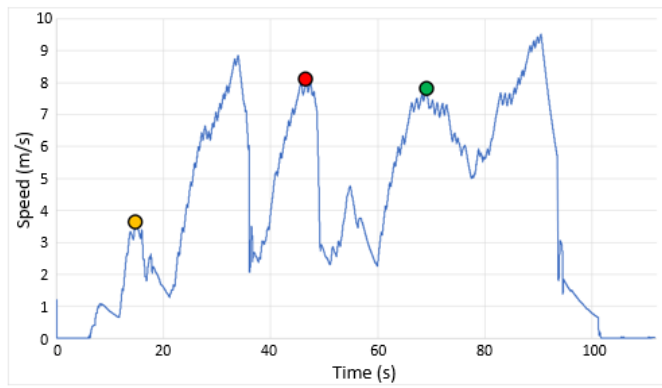

a)

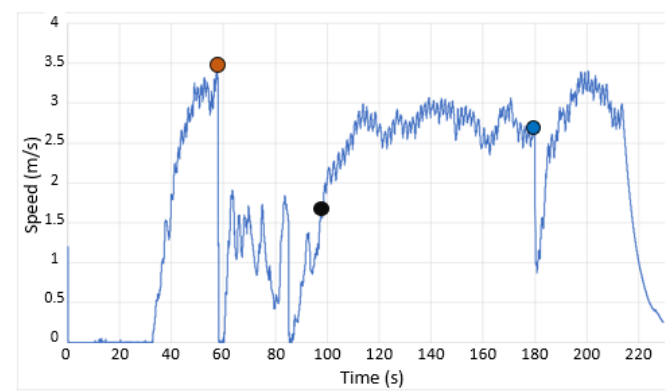

b)

Figure 12. Comparative results of speed data from two participants: a) participant P15 and b) participant P10 as a function of time in balance mode.

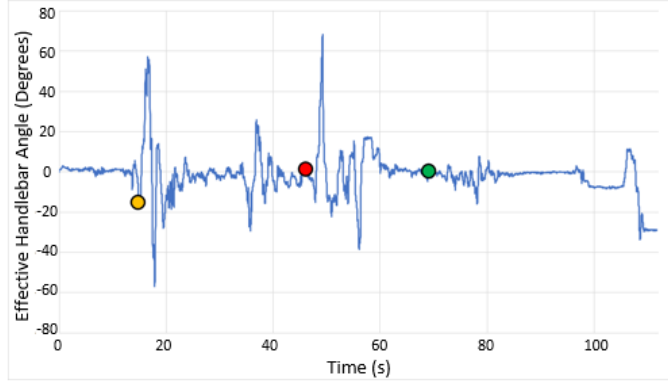

a)

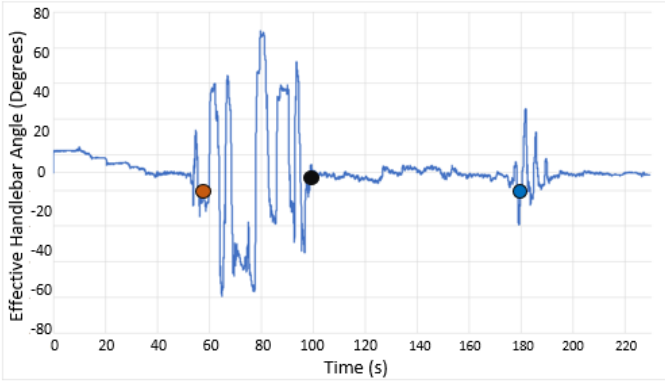

b)

Figure 13. Comparative results of steering angle data from two participants: a) participant P15 and b) participant P10 as a function of time, in balance mode.

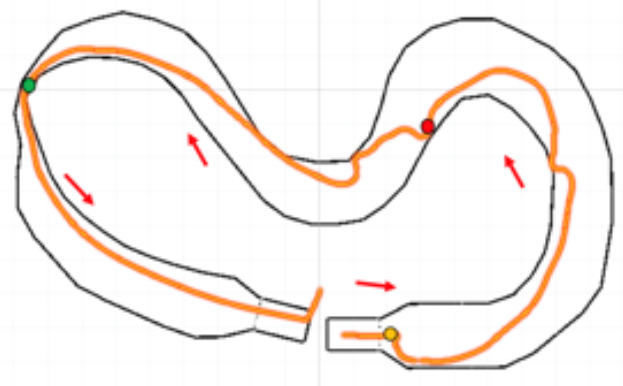

a)

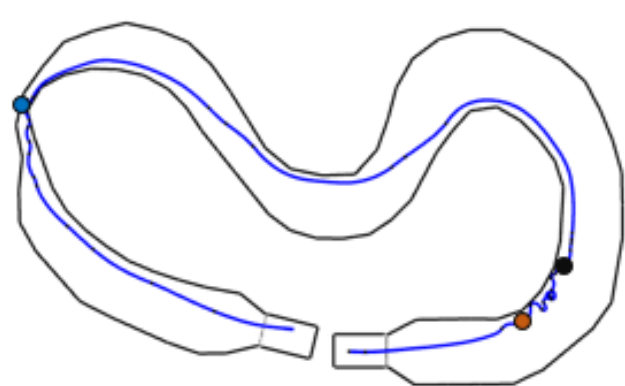

b)

Figure 14. Trajectory through the experimental track: P15 at a) and P10 at b).

perception, that contribute to the cyclist's comfort and immersion in the virtual environment, regardless of the mode used.

From the results obtained through the SimBike-specific questionnaire, we identified that the simulator (in both modes) was easier to use for the set of participants who first used the Balance mode and, after that they used Handlebar mode. They gave higher marks for "Ease of Use", "Ease of Steering" and "Steering realism" than the other group in both modes. In particular, the score they attributed to "Ease of Steering" indicates that they were quite impressed by the Handlebar mode (6.29 on the second experiment), suggesting that they had more difficulty steering in the Balance mode (4.25 on the first experiment).

It is worth noting that the real bicycle is not accelerating or moving. As a consequence, when exiting a turn, there is no force pushing the participant back to a balanced position, causing a strange sensation when the participant must balance itself back on it's own. Besides that, we can notice that the participants which had their first contact with VR in SimBike had no trouble using the simulator, indicating that the proposed simulator has characteristics that make it easy to use and accessible, since accelerating movement is usually very uncomfortable in VR.

One important sensory feedback modality associated with speed is the wind sensation. As the wind sensation and wind's contribution to immersion received both the highest grades, we can infer that this sensory device worked as expected, contributing to the immersion of the simulation.

Concerning the terrain contact sensation, participants declared that they felt the bicycle vibration as a result of the bicycle's contact with the ground. The structure raised to hold the bicycle also contributed since the real tire vibrated when rolling. Also, the motors installed under the seat and on the Handlebar contributed to those moments where the bike's wheels are not spinning, but the bicycle on the Virtual world was still moving, but maybe not on the intensity needed. Results suggest that, compared to the tire's vibration, the vibra- 
tion from the motor attached to the seat and at the Handlebar was too gentle.

Regarding the collision sensation, as we use the HMD at the experiments, the fact that you are looking at an object approach contributes to the expectation of crashing into it. About the hit feeling, those vibrations made by the motors attached to the seat and Handlebar were smaller than the bicycle trepidation, reducing the crash sensation. Also, as the weight fixed on those motors were too heavy to the motor, there was a small delay between the real crash and the crash sensation. One important aspect to perceive is that, due to the delay between the impact and the insufficient motor vibration, most of the impact sensation came from the observation of the user.

Still, the balance load cell sensitivity is an indicator of the need of taking the user's total weight into account for Balance mode, instead of relying on the variations under weight transfer. After consideration, we understand that the load cells signal variation is measuring not weight transfer in itself, but some factor of the torque applied longitudinally to the bicycle frame. In other words, taller people create more torque in the bicycle frame when leaning with the same body angle than a shorter person with the same weight, making it harder for them to have fine control of the device as built. This is also true for a heavier person compared to someone else of the same height. Taking different participant height and weight into consideration is paramount in any follow-up work since there is a strong correlation between these two variables.

In relation to studies in the literature, we highlight that one positive aspect of our solution is the reduced latency of steering due to the use of the HMD controller, even when associated with the balanced input, which solves safety issues found by Sun and Qing (2018) and Bolton et al. (2014). Considering the freedom of movement and the load cell readings, the change of direction combined by the weight distribution can provide to the user a sensation that his body is considered while changing directions. Also, centering problems of some mechanical devices as reported by Bolton et al. (2014) could be eliminated. Although the simulator developed by Boulanger et al. (2017) employed potentiometerbased brakes and wind feedback too, that work didn't evaluate the effectiveness of these devices.

Analyzing the IPQ questionnaire applied to the participants raised some facts that could contribute to the results obtained. Based on the high scores obtained in Spatial presence, regardless of the experiment mode, we can conclude that the combined devices and feedbacks created an immersive experience. The HMD device certainly contributed to the spatial presence, since it is capable of providing the perception of depth, improving the notion of space and velocity in a virtual environment.

Some aspects as the absence of an avatar and the uncomfortable position for shorter users could have interfered with better user experience. Thus, it is necessary to include more hardware configuration options for the bicycle, like adjustable seat levels and a higher handlebar.

Regarding the results obtained in the SSQ Questionnaire, there was no report on severe symptoms of cybersickness. Most cases are concentrated on mild symptoms. It is worth note that during movement in VR, there are conflicts between vision and the other senses (e.g., vestibular and proprioception) that can generate sometimes severe cybersickness. Based on those facts there is evidence that the added feedback by SimBike contributed with the comfort of the participants, reducing such conflicts where possible.

\section{Conclusions}

In this study, we present an experiment that involves the use of a bicycle as a sensorimotor device in a virtual tour capable of providing a pleasant experience for the user. We designed SimBike, a virtual bicycle simulator with low-cost equipment, instead of the commonly used motor devices (steering using the Handlebar).

For our experiment, a track was created to allow the user to explore the implemented features, analyzing their interaction with the equipment. For that, two modes were created (Balance mode and Handlebar mode), both diverging only in the bike control mode. After implementing these modes, we evaluated the simulator by observing the tests and applying three questionnaires. We address aspects related to the use of the developed devices, immersion of the simulator, and the discomfort generated when using them.

We found in our tests that the implementation of the acceleration proved to be satisfactory. Also, the Handlebar attenuation by bicycle speed offers good precision and latency. Its concepts sought to provide greater comfort to the user and greater involvement in the simulator.

Results observed in both modes indicate the existence of comfort and immersion characteristics in the virtual environment. We also found that the proposed simulator configuration was more favorable for short stature users, who reported higher immersion values, as well as identifying that, from the SSQ questionnaire, there was a small increase in intensity and symptoms in the Balance mode. This increase was observed mainly in the six participants with a height above $1.80 \mathrm{~m}$.

Regarding the results obtained in the IPQ questionnaire, we identified very similar values in both modes, but the assessments of the participants' perception of the situations generated by the devices in the simulator were better than those obtained in the IPQ.

Concerning user experience and ease of use, users with weights over $90 \mathrm{~kg}$ perceived greater sensitivity in the Balance mode as a result of greater readings on the factors to be increased on the Handlebar, however, we found that most participants highlighted in the think-aloud that cycling in the simulator was "very pleasant" and easy to use.

In the SimBike-specific questionnaire, we found that the users gave high grades to wind perception and contribution to immersion, indicating that this feedback modality is very important for this activity. Users also gave high scores for "Joyfulness", praising the overall experience.

Considering that the first contact with the simulator happened on that experiment and most participants had very little experience with VR we found that SimBike has characteristics that make it an accessible experience.

Our solution succeeds in allowing the leaning of the users' 
body to cause an expected change in the simulation, while mitigating possible frustrations and reducing gaps in the available sensory feedbacks, but the results obtained lead us to reflect on improvements to be made and new tests to assess the use of weight combined to the Handlebar as an input method.

Based on these reflections, there is the possibility of the following future works:

- Improve the balance's calibration algorithm and procedure.

- Track user's hand positions and possibly hand movements to link to the avatar's hands, thus increasing immersion. Implementations could rely on LeapMotion or wearing the HMD controllers for tracking only.

- Implement controllable variable resistance for pedaling, making it possible to simulate the effort required to climb a steep slope.

- Improvements in haptic response, reducing latency and better transmitting the sensation of contact with the ground.

- Improve immersion by adding more sound effects, such as the sound of the bicycle's chain and other events related to haptics feedback.

- Enhance the fan control system to compensate for it's acceleration latency.

Other prominent projects are the adaptation of work for rehabilitation as well as the use of cycling simulators for traffic and safety engineering.

\section{References}

Ahmadpour, N., Ijaz, K., Calvo, R., and Wang, Y. (2017). Physical activity enjoyment on an immersive vr exergaming platform. In IEEE Life Sciences - Multi-society perspectives on personalized healthcare and wearables, pages $13-15$.

Altmayer Sport (2019). Al04 - alt cicle - rolo de treinamento. https://www . altmayer. com.br/produtos/ver/40/ al04-alt-cicle-rolo-de-treinamento. Accessed: 2019-05-05

Arduino AG (2020). What is arduino? https://www arduino.cc/en/Guide/Introduction. Accessed: 2020-07-11.

Bolton, J., Lambert, M., Lirette, D., and Unsworth, B. (2014). Paperdude: A virtual reality cycling exergame. Conference on Human Factors in Computing Systems - Proceedings, pages 475-478.

Boulanger, P., Pournajib, A., Mott, W., and Schaeffer, S. (2017). A low-cost virtual reality bike for remote cardiac rehabilitation. In Barbic, J., D’Cruz, M., Latoschik, M. E., Slater, M., and Bourdot, P., editors, Virtual Reality and Augmented Reality, pages 155-166, Cham. Springer International Publishing.

Carvalho, M. R., da Costa, R. T., and Nardi, A. E. (2011). Simulator sickness questionnaire: Tradução e adaptação transcultural. In Jornal Brasileiro de Psiquiatria, volume 60, pages 247-252.
Chavarrias, M., Carlos-Vivas, J., Collado-Mateo, D., and Perez-Gomez, J. (2019). Health benefits of indoor cycling: A systematic review. Medicina, 55:452.

de Souza e Almeida, R., Cherullo, R., Radetic, D., Francy M. Silva-Calpa, G., and Barbosa Raposo, A. (2019). Exploring Motor and Sensory Devices in a Bicycle Simulator. In 201921 st Symposium on Virtual and Augmented Reality $(S V R)$, number November, pages 232-241. IEEE.

Freina, L. and Ott, M. (2015). A literature review on immersive virtual reality in education: State of the art and perspectives. In Proceedings of the 11th International Scientific Conference eLearning and Software for Education (eLSE), pages 133-141.

Fuchs, P., Burkhardt, J.-M., and Lourdeaux, D. (2011). Theoretical and pragmatic approach to virtual reality. In Fuchs, P., Moreau, G., and Guitton, P., editors, Virtual Reality: Concepts and Technologies, pages 12-15. CRC Press.

Kennedy, R. and Frank, L. (1985). A review of motion sickness with special reference to simulator sickness. Technical Report 81-C-0105-16, Naval Training Equipment Center, Orlando, FL.

O'Hern, S., Oxley, J., and Stevenson, M. (2017). Validation of a bicycle simulator for road safety research. Accident Analysis and Prevention, 100:53-58.

Pedroli, E., Greci, L., Colombo, D., Serino, S., Cipresso, P., Arlati, S., Mondellini, M., Boilini, L., Giussani, V., Goulene, K., Agostoni, M., Sacco, M., Stramba-Badiale, M., Riva, G., and Gaggioli, A. (2018). Characteristics, Usability, and Users Experience of a System Combining Cognitive and Physical Therapy in a Virtual Environment: Positive Bike. Sensors, 18(7):2343.

Pepper, N. (2019). The zen of communication. https: //natepepperdotcom. wordpress.com/2019/03/19/ virtual-reality-cycling-is-finally-here-and-it-is-am Accessed: 2020-07-11.

Rakhmatov, R., Abdulali, A., Hassan, W., Kim, M., and Jeon, S. (2018). Virtual reality bicycle with data-driven vibrotactile responses from road surface textures. In IEEE Games, Entertainment, Media Conference (GEM), pages 1-9.

Schubert, T., Friedmann, F., and Regenbrecht, H. (2001). The experience of presence: Factor analytic insights. Presence, 10:266-281.

Schulzyk, O., Bongartz, J., Bildhauer, T., Hartmann, U., Goebel, B., Herpers, R., and Reinert, D. (2007). A Bicycle Simulator Based on a Motion Platform in a Virtual Reality Environment - FIVIS Project. In Advances in Medical Engineering, pages 323-328. Springer Berlin Heidelberg, Berlin, Heidelberg.

Slater, M. and Wilbur, S. (1997). A framework for immersive virtual environments (five): Speculations on the role of presence in virtual environments. Presence: Teleoperators and Virtual Environments, 6(6):603-616.

Sun, C. and Qing, Z. (2018). Design and construction of a virtual bicycle simulator for evaluating sustainable facilities design. Advances in Civil Engineering, 2018:1-10.

Unity Technologies (2019). Unity core platform. https: //unity.com/products/core-platform. Accessed: 2019-07-15. 